\title{
Reflexiones acerca de la evaluación formativa en el contexto universitario
}

\author{
Thoughts on formative assessment in the university context
}

María Esther Hidalgo Apunte ${ }^{1}$

Recibido: 06.12.2020

Aceptado: 20.12.2020

\section{Resumen}

La evaluación formativa plantea nuevos desafíos a la educación universitaria, ya que en este contexto la evaluación aplicada a los estudiantes es mayoritariamente tradicional, controladora y sumativa. Lo que ha provocado perder el horizonte de la verdadera intención de la evaluación, la cual buscar plantear en el andar del proceso de enseñanza aprendizaje orientaciones y modificaciones a las estrategias pedagógicas para mejorar el aprendizaje de los alumnos/as, convertirlo en protagonista activo de su proceso de aprendizaje, y a que este entienda su desarrollo metacognitivo y lo regule.

Palabras clave: Evaluación formativa, Docente universitario

\begin{abstract}
Formative evaluation poses new challenges to university education, since in this context the evaluation applied to students is mostly traditional, controlling and summative. This has caused the loss of the horizon of the true intention of the evaluation method, which seeks to propose in the course of the teaching-learning process orientations and modifications to the
\end{abstract}

${ }^{1}$ mariaestherhidalgo@gmail.com 
pedagogical strategies aimed at improving the learning of the students, making it an active protagonist of their learning process, since it understands its metacognitive development and regulates it.

Keywords: Formative assessment, University teacher

\section{Introducción}

La evaluación de los aprendizajes constituye uno de los aspectos clave dentro del desarrollo de los procesos de enseñanza aprendizaje. Desde la perspectiva docente, la evaluación es el medio que permite valorar el progreso, los logros y el alcance de los objetivos de aprendizaje de cada alumno/a; pero además, es una herramienta útil que durante el ejercicio profesional y la reflexión acerca de la labor docente, ayuda a contrastar la adecuación de las metodologías didácticas adoptadas, lo que posibilita introducir mejoras en los diferentes elementos curriculares con el propósito de optimizar no sólo el quehacer docente, sino que de cara a los alumnos/as la evaluación formativa les permite a estos examinar su avance en cuanto a la asimilación de los contenidos y la adquisición de habilidades o competencias y representa una oportunidad de reorientación de su aprendizaje.

Tradicionalmente, la evaluación sirve a la educación superior como instrumento de control de los resultados obtenidos por el alumnado y está focalización en la asignación de calificaciones, las cuales determinan la superación de materias y por ende la obtención de una titulación, lo que conlleva a que los alumnos/as fijen su atención sobre los procesos de evaluación y procuran responder a las exigencias sumativas o de resultados de este proceso, dejando de lado su carácter formativo (Gill, 2012). Provocando como resultado la aplicación de una evaluación tradicional, controladora y poco formativa y la han dotado de un evidente carácter sumativo.

Sin embargo, durante las últimas décadas el interés que se ha dado al rediseño del proceso de enseñanza aprendizaje ha permitido la reconfiguración y valorización de la evaluación. La 
evaluación actualmente tiene un carácter formativo, y da una orientación efectiva a los estudiantes acerca de su proceso de aprendizaje. Sin embargo, y a pesar de esta nueva concepción acerca de la evaluación y sus grandes ventajas en favor del fortalecimiento del proceso de enseñanza aprendizaje, existe una insuficiente aplicación de la evaluación formativa por parte de los docentes universitarios.

Por ende, uno de los mayores temas educativos que genera preocupación y discusiones en el ámbito educativo es el de la evaluación de los aprendizajes. Y como ya se advirtió, la evaluación mayormente manifestada en las aulas es la tradicional, la cual no garantiza un aprendizaje significativo y sólo busca aprobar o desaprobar al alumno/a. En la actualidad, la educación universitaria ha de responder a las nuevas formas de enseñanza y de aprendizaje, así como también al mejoramiento de la evaluación de dichos aprendizajes.

Por ello, esta breve reflexión de la evaluación formativa aplicada por los docentes universitarios busca situar a este tipo de evaluación como una estrategia para la adquisición de nuevos saberes de forma más proactiva y para el desarrollo de habilidades y actitudes por parte de los alumnos/as, siendo el docente universitario la pieza clave, ya que son quienes figuran como protagonistas activos y consientes del manejo de la evaluación. Los docentes mediante la evaluación formativa recogen "los logros progresivos del aprendizaje en los estudiantes con el objeto de mejorar, corregir o reajustar el avance dentro del contexto socio cultural donde se desarrolla, ya que ésta determina la eficacia del acto didáctico en todo el proceso" (Ortega, 2015, pp. 9).

El carácter formativo de la evaluación es entonces uno de los ejes importantes de cambio de la concepción de la enseñanza universitaria y su implantación requiere una transformación cultural. Un cambio que deje al margen y se libere de presiones ante la obtención de resultados conseguidos por la evaluación tradicional como un indicio de calidad educativa. Ya que la evaluación formativa no persigue alcanzar metas relacionadas a la cultura de los resultados, la cual busca obtener datos para fines administrativos y económicos; la evaluación formativa 
pretende potenciar el logro de los aprendizajes de los alumnos/as, mejorar las prácticas de enseñanza aprendizaje y busca motivar al alumnado frente a su proceso de formación.

\section{Referentes}

La evaluación formativa es un proceso en el cual tanto alumnos/as como docentes comparten metas de aprendizaje. Y por el cual, el docente evalúa constantemente los avances en relación con los objetivos de aprendizaje que permitan alcanzar dichas metas; con el propósito de determinar y ajustar los métodos y las estrategias en función de fortalecer el proceso de enseñanza aprendizaje llevado a cabo.

Con respecto a ello, se han escrito numerosos estudios acerca de la validez de la evaluación formativa en la educación superior, estos abogan por procesos de evaluación transformadores, que formen al alumno/a de manera integral y holística (Zabalza, 2002: Álvarez, 2003; LópezPastor 2004; Bonsón y Águeda, 2005; Watt y García, 2006 entre otros). Además, existen estudios los cuales aluden a la evaluación formativa como un eje fundamental de articulación de todo el proceso de enseñanza aprendizaje (Brown y Glasner, 2003; Delgado, Borges, García, Oliver y Salomón, 2005; Villardón, 2006, entre otros).

Diversos autores sostienen también, que la evaluación formativa incide de manera significativa y positiva sobre el alumnado, ya que se sienten mucho más implicados con su proceso de aprendizaje, y que además contribuye la evaluación formativa al desarrollo de la autorregulación del proceso de aprendizaje Watt y García, 2006; López, 2009; Fernández, 2010, Morales, 2010; Castejón, López, Julián y Zaragoza, 2011).

Otros autores coinciden, en que la evaluación formativa mejora y optimiza los procesos de enseñanza aprendizaje llevados en la universidad (Dochy, Segers y Dierick, 2002; De la Fuente y Justicia, 2003, De Miguel Díaz, 2006; Martínez, Martín y Capllonch, 2009). 
Todos estos trabajos mencionados anteriormente, reconocen a la evaluación formativa como una herramienta y una ocasión para el aprendizaje; una herramienta que permite la construcción del aprendizaje, ya que la evaluación formativa está fundamentada en la teoría socio cultural y el constructivismo.

La teoría sociocultural propuesta por Vygotsky plantea que todo aprendizaje tiene su origen en un entorno social y destaca la importancia de los procesos interpsicológicos y de interacción social en el aprendizaje. Desde el planteamiento de esta teoría, el aprendizaje es un proceso beneficioso de transformación cognitiva y social que se da en un contexto colaborativo. Es decir, cada uno de los individuos aprendemos al observar e interactuar con otras personas; las relaciones que los individuos puedan establecer con otros individuos de más experiencia o mejor capacitados determinan el aprendizaje social y cultural, siendo el lenguaje durante estos procesos interpsicológicos y de interacción social la herramienta más importante y primordial para la realización e interiorización del conocimiento.

En este sentido Vygotsky da a entender, que los individuos no son pasivos, y que el contexto educativo debe ser un espacio ideal de desarrollo, a través de la comunicación efectiva de signos orales o escritos. Pero en sí, el contexto educativo es capaz de garantizar este desarrollo si ofrece "acompañamiento, ayuda, colaboración, orientación y guía por parte del docente, valiéndose de instrumentos didácticos, técnicas y estrategias metodológicas a través de diferentes tipos de actividades guiadas y controladas, por el que enseña hacia el que aprende (Ivic, 1994; citado por Ortega, 2015).

La educación y su contexto por consiguiente permite, un proceso de interacción socio cultural entre docente y estudiante, y cuyo resultado es la formación del hombre en una persona insertada en el contexto donde vive, como afirma Makarenko (1934; citado por Palacios, 2009). Esta relación continua docente - estudiante da paso a la formación, el aprendizaje y el desarrollo de habilidades por parte del estudiante que lo convierten en un ser humano inserto y activo en la sociedad. 
Así también, la relación docente - estudiante y tomando en cuenta otros de los postulados de Vygotsky, es una oportunidad de avanzar a la Zona de Desarrollo Próximo (ZDP), que en líneas generales representa la diferencia entre lo que el individuo ya puede realizar o hacer por sí sólo (lo adquirido) y lo que aún tiene que desarrollar con ayuda de otra u otras personas, es decir, es aquello que está en vías de ser adquirido.

Para el docente la ZDP debe ser un espacio creado para el estudiante, en el cual y partiendo de una serie de conocimientos previos por parte del estudiante, este puede llegar a resolver situaciones nuevas y realizar actividades complejas con eficacia, que en un inicio fueron difíciles para él, pero que gracias a este espacio interactivo denominado ZDP es capaz de llevarlas a cabo.

Se está en la Zona de Desarrollo Próximo de una persona que aprende sí, y solo sí, un mediador humano (docente) puede, con su intervención, reducir la demanda mental que requiere la solución del problema hasta el punto donde la solución se vuelve accesible a la persona que aprende.

Por consiguiente, resulta imprescindible que el docente construya o desarrolle la ZDP por medio de prácticas educativas que den paso a la aplicación de estrategias metodológicas activas y acertadas en el proceso de enseñanza aprendizaje. Es así, que la evaluación formativa no sólo es una herramienta idónea, sino que además es una práctica educativa que fortalece la enseñanza, dado que indica al estudiante el conocimiento o la destreza que no posee, pero que puede llegar alcanzar por medio de acciones específicas creadas a partir de una evaluación.

La evaluación formativa permite entonces, conocer la distancia entre el nivel actual de desarrollo y el nivel más avanzado de desarrollo potencial; la evaluación así concebida se convierte entonces en una práctica trascendental hacia la formación y el aprendizaje.

En consecuencia, la evaluación formativa define el campo de posibilidades del estudiante y el espacio o ZDP que ha de crear el docente, permitirá generar un ambiente didáctico motivador 
e interesante, con lo cual, el conocimiento previo del estudiante (nivel actual de desarrollo), se potencialice en otros nuevos y mejores saberes y conocimientos (nivel de desarrollo potencial).

De acuerdo con Ruiz (2009), el papel del docente es propiciar la creación de la Zona de Desarrollo Próximo para ayudar a adquirir un aprendizaje futuro e inmediato en el estudiante. Los conocimientos o habilidades previas que el estudiante posee pueden transformarse en nuevos y superiores saberes o conocimientos con la ayuda, guía, orientación e interacción adecuada por parte del docente y los compañeros, consiguiendo con ello que el estudiante pueda resolver realidades nuevas y actividades complejas con el apoyo y aplicación de la evaluación formativa.

El constructivismo en cambio, pero en similitud a la teoría socio constructivista plantea que nuestro mundo es producto de las interacciones humanas, y en tal magnitud el conocimiento no se recibe pasivamente, sino que es un proceso activo de construcción. "El sujeto que conoce y la función cognoscitiva está al servicio de la vida, es una función adaptativa y, en consecuencia, lo que permite el conocimiento al conocedor es organizar su mundo, su mundo experiencial y vivencial” (Flores, 1998, citado por Gonzáles y Hernández 2006).

En el constructivismo el alumno/a, es concebido como un individuo capaz de desarrollar su autonomía para convertirse en un individuo capaz de aprender a aprender mediante los procesos interpsicológicos y socio culturales que se dan en el ambiente educativo, y en los cuales el docente debe fomentar la participación activa del alumno durante el proceso enseñanza aprendizaje. Es así como, la evaluación formativa es una herramienta idónea para fomentar la participación activa y la interacción social, lo que la lleva a ser concebida como una evaluación constructivista, favoreciendo la evaluación de las experiencias propias del alumno y de sus vivencias como formas válidas de aprendizaje.

La evaluación formativa fundamentada en el constructivismo plantea las siguientes características: 
La evaluación constructivista, no sólo se interesa y toma en cuenta los productos finales y observables del proceso de enseñanza aprendizaje; la evaluación bajo esta perspectiva considera de gran importancia los procesos de construcción que dieron origen a estos productos, la organización y estructuración de las construcciones elaboradas. Díaz y Hernández (2002) citado por Gonzáles y Hernández 2006, señalan que las conductas que demuestran la ocurrencia de algún tipo de aprendizaje dan origen a todo un proceso de actividad constructiva (procesos y operaciones cognitivas) que finaliza en la elaboración de determinados tipos de representaciones (esquemas, significados, etc.) sobre los contenidos curriculares.

La evaluación constructivista le permite al docente valorar aspectos iniciales, así como aquellos que va manifestando el alumno/a durante el proceso de construcción del conocimiento. Es decir, está ligada a la Zona de Desarrollo Próximo propuesta por Vygotsky; lo que faculta al docente a centrar la actividad evaluativa de forma continua según cada etapa del proceso de enseñanza aprendizaje.

A la evaluación constructivista le interesa la promoción y valoración del aprendizaje significativo, por eso el docente, y según Alfaro (2000) debe dirigir el proceso evaluativo hacia la valoración de: el grado de construcción de interpretaciones valiosas que los alumnos hayan logrado de los contenidos curriculares, lo que hace referencia a la significatividad de los contenidos; el grado alcanzado para dotar de sentido y utilidad a dichas interpretaciones, es decir la funcionalidad de los contenidos adquiridos; el grado de control y responsabilidad que los alumnos han alcanzado frente a su propio proceso de aprendizaje, lo cual además hace referencia a su desarrollo personal.

La evaluación de los aprendizajes de forma constructivista exige procedimientos y técnicas diferentes, pero lo realmente importante de estas técnicas e instrumentos es la apreciación que se logre mediante la aplicación de los mismos en cuanto a la significatividad y la atribución del sentido logrado por el alumno/a en cuanto a lo aprendido y ante su proceso de enseñanza aprendizaje. 
Las características antes mencionadas hacen visible a la evaluación constructivista como formativa, por la cual el docente promueve y valora los aprendizajes significativos con la ayuda de actividades evaluativas, es decir, asignando a los alumnos/as tareas, actividades y procedimientos de evaluación que requieran por parte de los alumnos/a interpretaciones y la construcción de significados como producto de los aprendizajes alcanzados.

El docente en consecuencia es un mediador, ya que fomenta actividades didácticas encaminadas a que los alumnos/as reconozcan y valoren la utilidad de lo aprendido y lo que aprenden, lo amplíen y apliquen en su contexto. Además, que les permite desarrollar a los alumnos/as la capacidad de autoevaluación, necesaria para que los individuos aprendan a evaluar su proceso y el resultado de su aprendizaje.

Cuando la evaluación es constructivista se abre una puerta de participación a los alumnos/as ya que se toman decisiones en conjunto (docente - alumno) acerca del proceso de enseñanza aprendizaje. El docente facilita el aprendizaje del alumno, promoviendo su participación y contribuyendo a su desarrollo integral, planteándole la evaluación como una actividad continua, integral y retroalimentadora, mientras que el alumno/a se compromete con su aprendizaje, autoevaluándose, y evaluando a sus compañeros y al proceso.

Así y bajo este paraguas de fundamentos, la evaluación formativa, un término educativo acuñado por Scriven por primera vez en el año de 1967, deja ver el proceso de evaluación como un proceso que no sólo valora el resultado, sino que, además, le proporciona al docente información constante, oportuna e indispensable frente algún objeto de estudio por parte del alumno (Rosales, 2014).

Es definida la evaluación formativa, es un proceso mediante el cual, se hace un acopio de datos informativos, como resultado de las diversas acciones académicas programadas por el docente y emprendidas por los alumnos, referidas a los procesos cognitivos, motrices y actitudinales, los cuales inducen al análisis de estos para tomar una determinación apropiada 
hacia la mejora del proceso pedagógico Chadwick (1989; citado por Fernández, Torrealba, Pineda y Tejerina, 2007).

Conforme a Gonzáles (2012), la evaluación formativa en un sentido general es una acción planificada, organizada y sistemática de un hecho o fenómeno educativos que nos lleva a la valoración de las cualidades y características de una actividad académica, desprendiéndose de esta valoración juicios y reflexiones que se traducen en la toma de decisiones frente al objeto evaluado y el evaluador.

En este sentido, también plantean Deiros, Calderón y Hernández (2002), que la evaluación ha de realizarse de forma sistemática teniendo en cuenta las funciones de la misma que son: comprobación y acreditación de los contenidos adquiridos por parte del alumno/a, de retroalimentación, de motivación educativa y, por último, desarrolladora y formativa.

Bajo estas conceptualizaciones, la evaluación formativa es un proceso que ayuda al alumno/a aprender a aprender, por lo cual la evaluación va más allá de un enjuiciamiento del desempeño del alumno/a de manera ocasional, para convertirse en una actividad sistémica y continua que impulsa al mejoramiento de la calidad del aprendizaje, favoreciendo con ello el rendimiento de los alumnos/as y del proyecto educativo institucional.

En consecuencia y como lo señala Casanueva (2005), la finalidad de la evaluación formativa es ayudar a la regulación y autorregulación del proceso de aprendizaje, y en la toma de decisiones que faciliten el progreso de los alumnos.

De forma general, la razón de ser de la evaluación formativa es buscar e interpretar evidencias acerca del logro de los alumnos/as respecto a una meta. Permitiéndole con ella al docente identificar dónde se encuentran sus aprendices, conocer las dificultades que enfrentan en su proceso de aprendizaje y determinar qué es lo que corresponde hacer y hacia dónde hay que dirigirse. 


\section{La evaluación formativa en la educación superior}

Las iniciativas del profesorado universitario para adoptar la evaluación formativa aún distan de un ideal, y las causas más relevantes dentro del ámbito universitarios son: limitada formación de los docentes en el campo de la evaluación de los aprendizajes, dificultad para la construcción de los instrumentos de evaluación formativa, presión y predominio de la cultura de resultados y falta de claridad de los criterios de la evaluación formativa.

La aplicación de la evaluación formativa en el contexto universitario requiere superar estas barreras; requiere modificar no sólo la actitud de los docentes, sino también involucrar a otras instancias del sistema educativo superior. Para que pueda aplicarse la evaluación formativa es necesario entonces cambiar los paradigmas sobre el uso de la evaluación y la cultura evaluativa; demanda conocer sus principios y dar cabida a sus planteamientos en las aulas y dentro del sistema educativo.

Por ello, la evaluación formativa en el ámbito universitario ha de ser considerada parte intrínseca y permanente de todo proceso didáctico; siguiendo esa línea Lorenzana (2012) nos dice que, la evaluación formativa es la herramienta didáctica más apropiada para hacer posible la aprehensión, interiorización y desarrollo de los aprendizajes; porque se orienta a mejorar la adquisición de conocimientos y desarrollo de capacidades, habilidades, destrezas, y actitudes del estudiante durante el proceso educativo, que no son tomados en cuenta en la evaluación tradicional.

En el contexto universitario hay que "orientar la evaluación hacia un proceso formativo, en contrapartida a una evaluación sumativa y finalista, con un alumnado activo y responsable de sus aprendizajes" (Castejón, Fraile, López y Romero, 2013, pp. 24). De ahí que, la evaluación formativa y su aplicación en el aula universitaria es considerada como una guía y herramienta para ayudar a desarrollar y fortalecer los procesos de enseñanza aprendizaje. Proceso que además impulsa la corresponsabilidad entre alumnos/as y docentes, y le otorga al alumnado 
protagonismo activo y autónomo frente a su proceso y lo forma integralmente sobre la adquisición de habilidades y competencias.

En suma, la evaluación formativa articula a todo el proceso de enseñanza aprendizaje. Como los sostienen Bordas y Cabrera (2001), la evaluación formativa es una evaluación integradora e incide cualitativamente en la calidad de la enseñanza y en la formación de la persona.

Por estas razones, la educación universitaria se enfrenta a un nuevo escenario, trascendental para la educación a nivel superior, en donde el alumno/a se convierte en el centro del proceso de enseñanza aprendizaje, lo que conlleva a una verdadera restructuración de su sistema, y uno de sus principales objetivos es desarrollar sistemas e instrumentos de evaluación formativa que sean coherentes con estos nuevos escenarios.

Como lo expresan Ruiz, Ureña y Vallés (2011):

Tanto por coherencia curricular como por comprensión de los diferentes tipos de aprendizaje que se buscan, los procesos de evaluación van a sufrir también fuertes cambios; no sólo porque cambia el objeto de evaluación (de una serie de conocimientos, más o menos académicos, a una serie de competencias y conocimientos básicos y aplicados más complejos), sino también porque cambia profundamente el papel que puede y debe jugar la evaluación en la mejora de dichos procesos de aprendizaje. (p. 137)

Sin embargo, la limitada formación de los docentes como ya se advirtió anteriormente en el campo de la evaluación de los aprendizajes, la dificultad para la construcción de los instrumentos de evaluación formativa, la falta de claridad de los criterios de la evaluación formativa y la presión y el predominio de la cultura de resultados, son problemas o necesidades que tiene que hacer frente esta nueva concepción de la evaluación de los aprendizajes. 
Es evidente que todo ello implica un esfuerzo muy importante que afecta al conjunto de la organización universitaria y que no podrá llevarse a cabo sin promover cambios culturales y organizativos realmente significativos (Martínez, 2007). Parte de la promoción de estos cambios culturales, es la formación a los docentes en el campo de la evaluación formativa; el valor de la formación sobre los sistemas de evaluación, sus técnicas e instrumentos y sus criterios de evaluación, deben significar para las instituciones y organismos educativos parte de un proceso de perfeccionamiento profesional cuyo alcance involucra a la calidad de la educación, a la calidad de la gestión de los docentes y a los planteamientos de la innovación educativa.

Sin embargo, desafortunadamente aún muchas de las instituciones de educación superior no ofrecen la posibilidad de formación a los docentes en cuanto a la evaluación formativa, ya que "no basta con dar capacitación; hace falta el apoyo de otros cambios" (Martínez, 2013). La superación de las limitantes docentes en el campo de la evaluación formativa entonces no sólo es parte de un proceso de la mejora docente llevada a cabo por la universidad; es parte de un proceso que involucra a toda una comunidad educativa, y que requiere toma de decisiones acertadas para implementar y mejorar procesos más adecuados de capacitación tomando en consideración la realidad de los docentes, del centro educativo y el contexto.

En el contexto universitario, además se requiere reflexión acerca de la evaluación formativa tomando en consideración sus dimensiones, las cuales son:

- Herramienta didáctica y procesual que "tiene por objeto someter de manera sistemática y continua a revisión el plan inicial (diseño curricular) con el fin de reconducirlo en los términos que sean necesarios" (Castillo, 2003, pp. 93), lo que implica una interacción permanente entre el docente y el alumno/a, nutrida por el diálogo, la discusión y la reflexión generando con ello un aprendizaje dialógico.

- Herramienta reguladora que permite al docente gestionar de manera más eficaz y eficiente las actividades pedagógicas considerando no sólo los contextos sino a sus alumnos/as de forma individual y grupal, y lo faculta a regular de forma gradual las actividades 
formativas, la evaluación de los aprendizajes y el desarrollo de habilidades o competencias de los alumnos.

- Herramienta de aplicación continua, ya que la evaluación formativa es un trabajo pedagógico que sigue y está estructurado bajo secuencias didácticas ordenadas y progresivas que aproximan al alumno al objeto de evaluación (Elola y Toranzos, 2000).

- Herramienta retroalimentadora, con lo cual se posibilita la consolidación del aprendizaje por parte del estudiante, ya que significa por parte del docente transmitir de forma asertiva información al alumno acerca de su proceso de aprendizaje, sus aciertos y desaciertos.

- Herramienta innovadora, ya que permite que la evaluación se transforme de una evaluación de los aprendizajes a la evaluación para los aprendizajes y a la evaluación como aprendizaje (Pérez, 2012, pp. 231, 232). Ya que exige tanto a docentes como alumnos utilizar la creatividad, el pensamiento divergente de nivel superior, la imaginación y la invención para aprender y construir conocimiento.

Sin duda, que en ámbito universitario la evaluación formativa aporta elementos de juicio suficientes para la toma de decisiones en cuanto a las acciones educativas que han de emprender las instituciones de educación superior en relación con: la capacitación a los docentes en el ámbito de la evaluación formativa, las futuras estrategias de enseñanza aprendizaje y de la evaluación de este proceso considerando a la evaluación formativa como el eje central. Determinar estas acciones educativas concierne no sólo a docentes sino también a alumnos/as y autoridades, es decir a toda una comunidad educativa en post de la mejora de la calidad.

\section{Conclusiones}

Tras haber realizado esta breve reflexión acerca de la aplicación de la evaluación formativa en las aulas de educación superior podemos afirmar, que un claro principio de la evaluación formativa es el de concederle al alumno/a el rol protagónico y activo en todos los momentos que estructuran su proceso. Lo que implica, que el alumno/a es capaz de reconocer su proceso 
metacognitivo y sus capacidades, para finalmente evaluar sus logros y su desempeño en función de una mejora.

El docente universitario entonces, para lograr este fin brinda al alumno una serie de apoyos durante todo el proceso de enseñanza aprendizaje, y la evaluación formativa le permite planificar, estructurar sus clases y evaluar el proceso de aprendizaje de cada alumno/a frente al objeto de estudio, previendo la interacción de todos los alumnos, cuidando que todos tengan oportunidad de participación. En este sentido, el aula de clase da cabida a un ambiente de diálogo abierto, donde el docente actúa como guía y son los estudiantes a través de las actividades planificadas y estructuradas quienes construyen un aprendizaje activo, a la vez que adquieren conciencia de su proceso y autorregulan su aprendizaje.

Mediante la evaluación formativa la práctica docente se ve enriquecida ya que no sólo la información obtenida permite al docente reorientar o modificar sus estrategias pedagógicas, sino que, puede determinar cómo apoyar a sus alumnos, reflexiona sobre su práctica y sobre los recursos que tiene, y así puede tomar decisiones respecto de cómo seguir para obtener mejores resultados. En suma, la evaluación formativa fortalece de forma significativa el proceso de enseñanza aprendizaje y para que esta cumpla con su propósito tiene que situársela e iniciarse contestando las siguientes preguntas: ¿hacia dónde vamos?, ¿dónde estamos? y ¿cómo podemos seguir avanzando?

La consolidación de la evaluación formativa en la práctica profesional universitaria significa la construcción de instrumentos de evaluación por parte de los docentes; ya que son estos quienes fijarán los criterios de evaluación formativa de acuerdo con los objetivos de aprendizaje que se deseen conseguir, en alineación con los estándares de calidad educativa que se exigen dentro de un marco global, frente a lo cual si los docentes universitarios no están capacitados, el docente puede llegar a plantear actividades y construir instrumentos con criterios poco fiables y generar sentimientos de ineficacia, lo que puede generar el fracaso del sistema de evaluación a mediano o largo plazo. 


\section{Referencias}

Alfaro, M. (2000). Evaluación del aprendizaje. Maracaibo: IUPEL

Álvarez, J. (2003). La evaluación a examen. Ensayos críticos. Madrid: Miño y Dávila Editores.

Álvarez, M., y Ortúñez, P. (2011). Una experiencia de Evaluación Formativa en Historia Económica. 1-9.

Bonsón, M., y Águeda, B. (2005). Evaluación y Aprendizaje. En Águeda Benito y Ana Cruz (Eds.), Nuevas claves para la docencia universitaria en el Espacio Europeo de Educación Superior (pp. 87-100). Madrid: Narcea.

Bordas, M., y Cabrera, F. (2001). Estrategias de evaluación de los aprendizajes centrados en el proceso. Recuperado de https://dpegp.files.wordpress.com/2012/04/lectura-1-evaapren.pdf

Bretones, Antonio (2006). La participación del alumnado en la evaluación de sus aprendizajes. Sevillla: Kikiriki.

Brown, S., y Glasner, A. (2003). Evaluar en la universidad: problemas y nuevos enfoques. Madrid: Narcea Ediciones.

Casanueva, P. (2005). Evaluación educacional formadora. Recuperado el 27 de marzo de 2006 en http://www.monografias.com/trabajos14/evaeduca/evaeduca.shtml

Castejón, F., López, V., Julián, J., y Zaragoza, J. (2011). Evaluación formativa y rendimiento académico en la formación inicial del profesorado de educación física. International Journal of Medicine and Science of Physical Activity and Sport, 11 (42), 238-346. 
Castejón, J., Fraile, A., López, V., y Romero, R. (2013). La evaluación formativa en docencia universitaria y el rendimiento académico del alumnado. Recuperado de: https://dialnet.unirioja.es/descarga/articulo/4239063.pdf

Castillo, S. (2003). Vocabulario de evaluación educativa. Madrid: Pearson Educación, S.A.

De la Fuente, J., y Justicia, F. (2003). Regulación de la enseñanza para la autorregulación del aprendizaje en la Universidad. Aula abierta, 82, 161-171.

De Miguel Díaz, M. (2006). Metodologías para optimizar el aprendizaje. Segundo objetivo del Espacio Europeo de Educación Superior. Revista interuniversitaria de formación del profesorado, 20 (3), 71-92.

Deiros B., Calderón M. y Hernández L. (2002). Apuntes sobre didáctica de la matemática para ingeniería. Recuperado el 27 de marzo de 2006 en http://www.monografias.com/trabajos11/monogrr/monogrr.shtml\#eval

Delgado, A., Borge, R., García, J., Oliver, R., y Salomón, L. (2005). Competencias y diseño de la evaluación continua y final en el Espacio Europeo de Educación Superior. Programa de Estudios y análisis de la Dirección General de Universidades del Ministerio de Educación y Ciencia. Recuperado de http://campus.usal.es/ ofeees/ARTICULOS/competencias_evaluacion_eees_mec.pdf

Dochy, F., Segers, M., y Dierick, S. (2002). Nuevas vías de aprendizaje y enseñanza y sus consecuencias: una nueva era de evaluación. Revista de Docencia universitaria, 2 (2). Recuperado de http://revistas.um.es/redu/article/view/20051

Dunn, K.E. y Mulvenon, S.W. (2009). A critical review of research on formative assessments: The limited scientific evidence of the impact of formative assessments in education. Practical 
Assessment Research \& Evaluation, 14(7). Recuperado de: http://pareonline.net/getvn.asp?v=)14\&n=7

Elola, N., y Toranzos, L. (2000). Evaluación educativa: una aproximación conceptual. Recuperado de: http://www.oei.es/calidad2/luis2.pdf

Fernández, A. (2010). La evaluación orientada al aprendizaje en un modelo de formación por competencias en la educación universitaria. REDU. Revista de Docencia Universitaria, 8 (1), 11.

Fernández, F., Torrealba, J., y Pineda, O. (2007). Una propuesta metodológica para la evaluación del proceso de enseñanza-aprendizaje. Innovaciones de Negocios, IV (2), 307-404. Recuperado de: http://www.web.facpya.uanl.mx/rev_in/Revistas/4.2/A8.pdf

Gill, J. (2012). La evaluación del aprendizaje en la universidad según la experiencia de los estudiantes. Recuperado de https://www.unav.edu/publicaciones/revistas/index.php/estudiossobre-educacion/article/viewFile/2076/1941

Gonzáles, M. y Hernández, A. (2006). El constructivismo en la evaluación de los aprendizajes del álgebra lineal. Recuperado de https://www.redalyc.org/html/356/35617701016/

González, M. (2012). La evaluación formativa y la evaluación por competencias. La Habana: Editorial Universitaria. Recuperado de: http://revistas.mes.edu.cu

Hernández, R., Fernández, C., y Baptista, P. (2014). Metodología de la Investigación (Sexta ed.). México: Mc Graw-Hill Interamericana Editores.

Knight, P. (2005). El profesorado de educación superior. Formación para la excelencia: Madrid: Narcea. 
López Pastor, V., González, M. y Barba, J. (2005). La participación del alumnado en la evaluación: la autoevaluación, la coevaluación y la evaluación compartida. Tándem, 17, pp. $21-37$.

López Pastor, V., Vallés, C. y Monjas, R. (2009). La evaluación formativa en el proceso de convergencia hacia el Espacio Europeo de Educación Superior. Segovia: Escuela Universitaria de Magisterio de Segovia. Universidad de Valladolid.

López Pastor, Víctor. (2004). La participación del alumnado en los procesos evaluativos: la autoevaluación y la evaluación compartida en educación física. Didáctica de la Educación Física: una perspectiva crítica y transversal (pp. 265-290). Madrid: Biblioteca Nueva.

López Pastor. V. (2009). Evaluación formativa y compartida en Educación Superior: propuestas, técnicas, instrumentos y experiencias. Madrid: Narcea Ediciones.

López, V. (2012). Evaluación formativa y compartida en la Universidad: clarificación de conceptos y propuestas de intervención desde la Red Interuniversitaria de Evaluación Formativa. Psychology, Society, \& Education 4. 1, pp. 117-130. Recuperado de http://www.psye.org/articulos.php?id=86

Lorenzana, R. (2012). La evaluación de los aprendizajes basados en competencias en la enseñanza universitaria. Recuperado de https://d-nb.info/1029421889/34

Marasinghe, M. G., Meeker, W. Q., Cook, D., y Shin, T. (1996). Using graphics and simulation to teach statistical concepts. The American Statistician, 50, 342-351

Martínez, F. (2013). Dificultades para implementar la evaluación formativa. Recuperado de http://www.scielo.org.mx/scielo.php?script=sci_arttext\&pid=S0185-26982013000100009 
Martínez, L., Martín, M. y Capllonch, M. (2009). Una experiencia de desarrollo profesional del docente universitario de Educación Física a través de una práctica crítica, reflexiva y colaborativa. Cultura y Educación, 21 (1), 95-106.

Martínez, M. (2007). Reflexiones sobre aprendizaje y docencia en el actual contexto universitario. La promoción de equipos docentes. Recuperado de http://www.revistaeducacion.mec.es/re2008/re2008_09.pdf

Melmer, R., Burmaster, E., y James, T.K. (2008). Attributes of effective formative assessment. Washinghton, DC: Council of Chief State School Officers

Morales, P. (2010). Investigación e innovación educativa. REICE: Revista Electrónica Iberoamericana sobre Calidad, Eficacia y Cambio en Educación, 8 (2), 47-73.

Moreno, T. (2011). La cultura de la evaluación y la mejora de la escuela. Recuperado de http://www.scielo.org.mx/scielo.php?script=sci_arttext\&pid=S0185-26982011000100008

Ortega, M. (2015). Evaluación Formativa aplicada por los docentes del Área de Ciencias, Tecnología y Ambiente en el Distrito de Hunter. Arequipa. Recuperado de http://repositorio.upch.edu.pe/bitstream/handle/upch/118/Evaluaci\%C3\%B3n.formativa.apli cada.por.los.docentes.del.\%C3\%A1rea.de.Ciencia.Tecnolog\%C3\%ADa.y.Ambiente.en.el.di strito.de.Hunter.Arequipa.pdf? sequence $=3 \&$ is Allowed $=\mathrm{y}$

Palacios, L. (2009). El desarrollo de las capacidades como estrategia de aprendizaje. Asociación Educativa Vygotsky, 1-7.

Pérez, Á., Julián, j., López Pastor, V. (2009). Evaluación formativa y compartida en el Espacio Europeo de Educación Superior (EEES). Evaluación formativa y Compartida en Educación Superior. Madrid: Narcea, 19-43.

Pérez, I. (2012). Educarse en la era digital. Madrid: Ed. Morata. 
Rosales, M. (2014). Proceso evaluativo: evaluación sumativa, evaluación formativa y Assesment su impacto en la educación. Buenos Aires. Recuperado de http://www.oei.es/congreso2014/memoriactei/662.pdf

Ruiz, E., Ureña, N., y Vallés, C. (2011). La Evaluación Formativa en Docencia Universitaria. Resultados globales de 41 estudios de caso. Recuperado de http://redu.net/redu/files/journals/1/articles/197/public/197-203-2-PB.pdf

Ruiz, M. I. (2009). La evaluación basada en competencias. 1-22. Recuperado de http://www.cca.org.mx/profesores/congreso_recursos/descargas/mag_comp etencias.pdf

Villardón, L. (2006). Evaluación del aprendizaje para promover el desarrollo de competencias. Educación Siglo XXI, 24, 57-76.

Von Glasserfeld, E. (1987). Learning as a constructive activity. En C. Janvier (Ed.), Problems of representation in the teaching and learning of mathematics (pp. 3-17). Hillsdale, NJ: Erlbaum

Watts, F. y García, A. (2006). La evaluación compartida: investigación multidisciplinar. Valencia: Universidad Politécnica de Valencia.

Yuste, R. (2013). Una evaluación innovadora como factor de mejora de la enseñanza on-line. (Tesis Doctoral de la Universidad de Extremadura, España). Recuperado de http://dehesa.unex.es/handle/10662/440

Zabalza, M. (2002). La enseñanza universitaria: el escenario y sus protagonistas. Madrid: Narcea Ediciones. 
REVISTA INTERNACIONAL DE PEDAGOGÍA E INNOVACIÓN EDUCATIVA |

Volumen 1. Número 1. Enero - Junio 2021

ISSN: 2745-0341 (En línea) 